\title{
Localization of the sea urchin Spec3 protein to cilia and Golgi complexes of embryonic ectoderm cells
}

\author{
Elizabeth D. Eldon, ${ }^{1}$ Isabelle C. Montpetit, ${ }^{2}$ Thomas Nguyen, ${ }^{1}$ Glenn Decker, ${ }^{1}$ \\ Maria C. Valdizan, ${ }^{1}$ William H. Klein, ${ }^{1}$ and Bruce P. Brandhorst ${ }^{2,3}$ \\ ${ }^{1}$ Department of Biochemistry and Molecular Biology, The University of Texas M.D. Anderson Cancer Center, Houston, Texas \\ 77030 USA; ${ }^{2}$ Department of Biology, McGill University, Montreal PQ H3A 1B1 Canada
}

\begin{abstract}
Expression of the Spec3 gene of Strongylocentrotus purpuratus is associated with ectodermal ciliogenesis. An antiserum was raised against the amino terminus of the deduced Spec 3 amino acid sequence and used for immunofluorescent staining. Cilia and an apical structure at the base of the stained cilium of each ectodermal cell stained intensely in gastrula and later stage embryos. Microtubule-depolymerizing agents dispersed the concentrated spot of apical staining, suggesting a localization of Spec3 antigen to the Golgi complex. Immunogold electron microscopy confirmed the localization of Spec3 antigen on cilia and in the Golgi complex. Spec3 antigen showed a diffuse punctate staining pattern in the ectodermal cytoplasm of hatching blastula when Spec3 transcripts are most prevalent, suggesting that after synthesis, Spec 3 is sequestered in the Golgi complex before appearing on cilia. Whereas the predicted $M_{\mathrm{r}}$ of the Spec3 protein is 21,600, immunoblotting with $S$. purpuratus proteins indicated that a Spec3 antigen was concentrated in cilia and migrated as an SDS-resistant aggregate of $M_{r} \sim 350,000$. Spec3 is also concentrated in cilia of Lytechinus pictus but the protein migrated with an $M_{r} \sim 23,000$ in this species. The $S$. purpuratus Spec3 antigen remains associated with the ciliary axoneme after extraction of membrane proteins.
\end{abstract}

[Key Words: Spec3; cilia; Golgi complex; sea urchin embryos; embryonic ectoderm]

Received August 28, 1989; revised version accepted October 31, 1989.

Ciliogenesis is an early and distinct morphological event in sea urchin development. It marks the end of the initial, rapid cleavage stages and coincides with the breakdown of the synchrony of cell division (Masuda and Sato 1984). The timing of individual cilium assembly depends on the position of the particular blastomere along the animal-vegetal axis (Masuda 1979); the blastomeres in the animal hemisphere, which give rise exclusively to ectodermal derivatives, are first to form cilia, at the eighth or ninth cleavage. One cleavage cycle later, the blastomeres in the vegetal hemisphere, which give rise to both ectodermal and endodermal derivatives, form their cilia. The process of ciliogenesis requires the coordinate assembly of a large number of different proteins that make up the structural elements of the cilium, including the axoneme, ciliary membrane, basal body, and rootlet (Luck et al. 1982). However, except for $\alpha$ - and $\beta$ tubulin (Raff 1975), dynein (Ogawa and Gibbons 1976), and tektins (Linck and Stephens 1987), little is known about the proteins that are involved in the construction of cilia during embryogenesis.

Microscopic and histological evidence indicates that not all sea urchin cilia have the same structural charac-

${ }^{3}$ Current address: Institute of Molecular Biology and Biochemistry, Simon Fraser University, Barnaby British Columbia V5A 1S6 Canada. teristics and probably do not all perform the same function. The beating of ectodermal cilia propels the embryo, but ectodermal cilia at the animal pole, the apical tuft cilia, are longer than other cilia, do not beat, and may have a sensory function (Burke 1978, 1983). This is also thought to be the case for certain cilia on the oral surface of late-stage embryos (Nakajima 1986a,b). The cilia on the invaginating endoderm cells become luminal cilia of the gut and presumably assist food passage. During larval development, the pattern of surface cilia becomes quite elaborate, with several rows of ciliated cells and numerous axons composing ciliated bands, the first of which appears around the periphery of the oral surface (Burke 1978).

These complex and varied structures suggest that some ciliary proteins may have evolved to perform specialized functions exclusively for cilia of particular differentiating cell types of the embryo. Characterizations of novel ciliary proteins of embryos should aid in understanding cilia structure, function, and assembly during embryogenesis.

In a previous study, we characterized a gene, Spec3, whose expression is associated with the process of ectodermal ciliogenesis in Strongylocentrotus purpuratus (Eldon et al. 1987). The Spec3 message displays a temporal and spatial pattern of accumulation expected for a 
message whose translation product is involved in ciliogenesis. Furthermore, the accumulation profile for Spec3 mRNA is very similar to that of the sea urchin $\beta$-tubulin mRNAs characterized by Harlow and Nemer (1987) and known to be involved in ciliogenesis. Further evidence for involvement of Spec3 in ciliogenesis was provided by changes in Spec3 message levels in deciliated and ciliaregenerating embryos. Deciliation results in a rapid fourto fivefold increase in Spec3 message levels, similar to the increase in tubulin mRNAs in sea urchin embryos (Gong and Brandhorst 1987) and Chlamydomonas (Keller et al. 1984) after deciliation or deflagelation.

The Spec3 protein has not been identified in embryos, but the reading frame of the Spec 3 mRNA encodes a 21,600 dalton protein of unusual hydrophobicity and high proline content, lacking substantial sequence similarities to any known proteins (Eldon et al. 1987). To demonstrate the relationship between the Spec3 protein and cilia, we generated an antibody against the amino terminus of the Spec 3 reading frame. Here, we report on the use of this antibody to investigate the embryonic and cellular location of the Spec 3 protein, as well as some of its structural characteristics. Spec 3 is present on ectodermal, but not endodermal, cilia and is concentrated in the Golgi complex. Immunoblot analysis demonstrated that the Spec3 antigen has an electrophoretic mobility corresponding to a huge aggregate, which remains associated with the ciliary axoneme after extraction of membrane proteins from cilia. In Lytechinus pictus, the Spec3 antigen is also concentrated in cilia, but its mobility suggests that it disaggregates to the monomer form in this species.

\section{Results}

Generation of an antiserum against the Spec3 protein

The structural features of the putative Spec3 protein, deduced from the open reading frame of the Spec 3 mRNA, are unusual in several respects. Acidic and basic amino acids are rare (only 12 of 208 amino acids), with 11 clustered in short hydrophilic regions bounding two long hydrophobic domains of nonpolar amino acids in the carboxy-terminal half of the protein (Fig. 1; Eldon et al. 1987). The proline content of the protein (14\%) is unusually high, and the proline distribution asymmetrical. All 29 prolines occur within the amino-terminal 138 residues, 23 within the first 63 . The polar amino acids glutamine, asparagine, glycine, and tyrosine are also common in the amino-terminal half of the protein (Fig. 1).

In an effort to produce an antiserum against Spec3, we prepared a synthetic peptide corresponding to the amino terminus, which is generally high in immunogenicity (Walter and Doolittle 1983). A peptide corresponding to the first 14 amino-terminal residues of Spec3 was synthesized, linked to hemocyanin, and injected into rabbits. The induced antiserum was strongly reactive against the synthetic peptide and, with immunoblotting, showed a reaction with extracts of sea urchin embryonic

10

20

$30 \quad 40$

M A Q V APIT G APT D* PPA YPPPPQQAPPPQQPG Y G QPQL G Y G

$50 \quad 60 \quad 70 \quad 80$

QPPPQ L G G QPPPQL G Y G YPPP®ON N M M M N T V V V TAPA

$90 \quad 100 \quad 110 \quad 120$

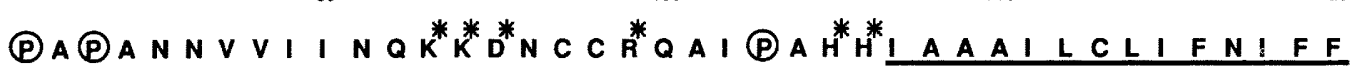

$130 \quad 140 \quad 150 \quad 160$

PGIGTIIAGFAVFCCGNPGA DGGS K

$170-180 \quad 190 \quad 200$

GTVWFFFLGWIWSIMWGAAFIGMSA DY HSGG DTTIVATGG

208

G G T T V I N N

Figure 1. Spec3 protein sequence deduced from Spec3 cDNA clone. Boxed region indicates the synthetic peptide used to generate Spec3 antibodies; asterisks indicate acidic or basic amino acids; proline residues are circled. The underlined residues represent the two hydrophobic domains discussed in the text, and the double-underlined Asn-Asn-Thr residues indicate the putative amino-linked glycosylation site. 
proteins (see below). The whole antiserum and the affinity-purified $\operatorname{IgG}$ fraction used in these investigations were also reactive against whole or sectioned embryos fixed by several methods. The distinctive pattern of staining obtained with this antiserum is described first.

\section{The distribution of Spec3 protein in gastrula and prism-stage embryos}

Gastrula- or prism-stage embryos were fixed briefly in methanol and processed for immunofluorescent staining with the Spec3 antiserum. At both stages of development, a regular punctate pattern of brightly stained structures was observed in ectodermal cells; a representative gastrula-stage embryo is shown in Figure $2 \mathrm{~A}$. Staining of the remainder of the ectoderm cell cytoplasm and endoderm cells was faint and comparable to the low level of background staining observed for preimmune antiserum (Fig. 2C). A prism-stage embryo that was immunostained for Spec3 is shown in Figure 3A at higher magnification. The brightly stained structures each appear to consist of a cluster of particles or vesicles. Cilia also stained intensely (Fig. 3B); focusing through the cell indicated that one of the brightly stained structures was at the base of each stained cilium. The cilia stained with a beaded pattern, suggesting that the Spec3 antigen is not uniformly distributed along their length. These observations indicate that Spec3 protein is associated with cilia and structures near the apical surface of ectodermal, but not of endodermal, cells. The possible identity of such structures included the basal body, ciliary root, and the Golgi complex.
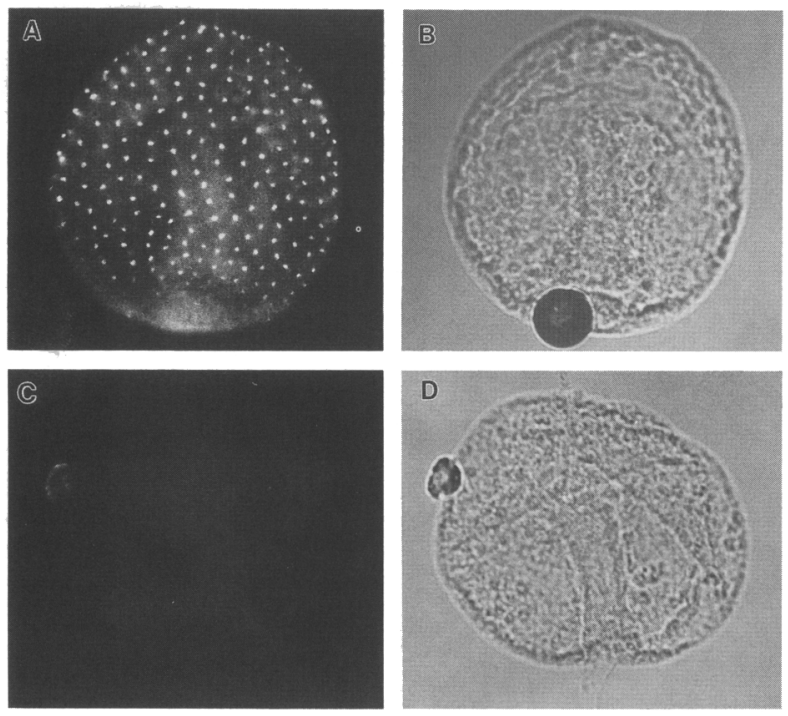

Figure 2. Spec3 protein distribution in gastrula-stage embryos. (A) Immunofluorescent image of an embryo labeled with Spec3 antiserum; the diameter of the embryo is $\sim 100 \mu \mathrm{m}$. $(B)$ Brightfield image corresponding to $A$. The dark spot at the bottom of the embryo is an air bubble. $(C)$ Embryo labeled with preimmune serum. $(D)$ Bright-field image corresponding to $C$.
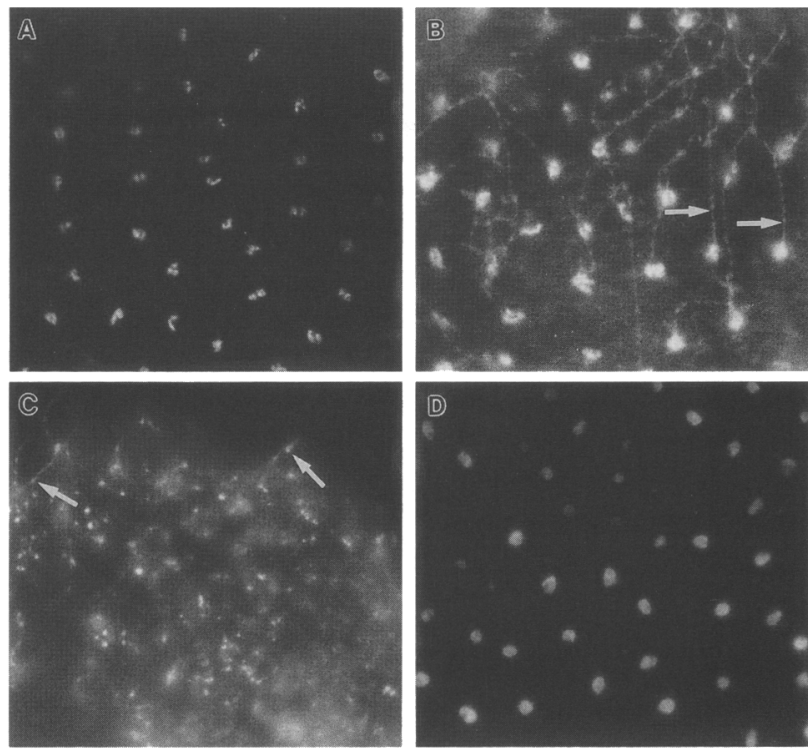

Figure 3. Distribution of $\mathrm{Spec} 3$ protein in prism-stage embryos treated with colcemid or monensin. Embryos were labeled with Spec3 antiserum and viewed under a $100 \times$ oil-immersion objective (resulting in a fourfold higher magnification than in Fig. 2). (A) The plane of focus was just below the ectodermal surface of the embryos, showing the brightly staining apical structures. $(B)$ The plane of focus was closer to the cell surfaces than that in $A$, showing the beaded staining pattern of cilia (arrows), as well as associated apical structures. $(C) \mathrm{Em}$ bryos were treated with $5 \mu \mathrm{M}$ rolcemid for $2 \mathrm{hr}$ before fixation. Cilia, shown by arrows, continued to stain with a beaded pattern. $(D)$ Embryos were treated with $10 \mu \mathrm{M}$ monensin for $2.5 \mathrm{hr}$ before fixation.

\section{Evidence that Spec3 is concentrated in the Golgi apparatus}

In many mammalian cells the Golgi complex has a polarized location near the centrosome, but disruption of microtubules results in dispersal of the stacks of cisternae throughout the cytoplasm (Lin and Queally 1982; Lipsky and Pagano 1985; Allan and Kreis 1986; Lucocq and Warren 1987; Miller et al. 1988). Prism-stage embryos were treated with colcemid at a concentration sufficient for disruption of microtubules of the cytoskeleton and spindles but not of the ciliary axoneme or basal bodies. As shown in Figure $3 \mathrm{C}$, the regular staining pattern of discrete apical structures was grossly altered: Ectodermal cells showed a fine punctate pattern of cytoplasmic staining, whereas the beaded pattern of staining of the cilia was unaltered (Fig. 3C). The distribution of Spec3 antigen was similarly disrupted in embryos treated with another microtubule-depolymerizing agent, nocodazole, but there was no obvious alteration in the staining pattern of embryos treated with the microfilament-depolymerizing agent cytochalasin B /data not shown). The alteration in the pattern of Spec3 staining was strikingly similar to that observed for the Golgi complex in cultured mammalian cells treated with microtubule-depolymerizing agents (Lin and Queally 1982; 
Lipsky and Pagano 1985; Allan and Kreis 1986), suggesting that Spec3 is localized to the ectodermal Golgi apparati, which are anchored in place by microtubules emanating from the basal body.

The sodium ionophore monensin prevents secretion of proteins via the Golgi complex in mammalian cells, resulting in the distension of the cisternae (Tartakoff 1983). Figure 3D shows the surface of an embryo treated with monensin for $2.5 \mathrm{hr}$. The stained structures were smoother and more regular in shape and were somewhat larger and more brightly stained than those in untreated embryos. With time, the staining of cilia decreased, becoming almost undetectable after $2.5 \mathrm{hr}$ of treatment. Similar results were obtained using concentrations of monensin over the range of $10^{-7}$ to $10^{-5} \mathrm{M}$. These results are consistent with the localization of Spec3 to the Golgi complex. The altered pattern of staining is probably the result of the distension of the Golgi, and the decline in staining in the cilia could be the result of a block in the transport of Spec3 protein from the Golgi complex to the cilium; this interpretation implies that Spec3 protein turns over rapidly on the cilium.

The distribution of stained cilia and Golgi complexes was consistent with there being one of each per ectoderm cell. Figure 4 shows the pattern of Spec3 staining in an embryo counterstained with ethidium bromide: Each stained Golgi complex and associated cilium was associated with a nucleus stained bright orange. Through-focusing indicated that nearly all ectodermal nuclei were associated with a stained apical Golgi complex and cilium (see also the staining of the ciliated band described below). Thus, the Spec3 antigen is expressed in virtually all ectoderm cells.

Several observations indicate the specificity of the Spec3 antiserum. In all experiments reported, an IgG fraction was used that had been stripped of activity against the keyhole limpet hemocyanin $(\mathrm{KLH})$ protein used for conjugation of the Spec3 synthetic peptide (see Methods). The stripped antiserum reacted actively with the Spec3 peptide in ELISA assays but weakly with KLH. Stripping eliminated the staining of a series of sea urchin

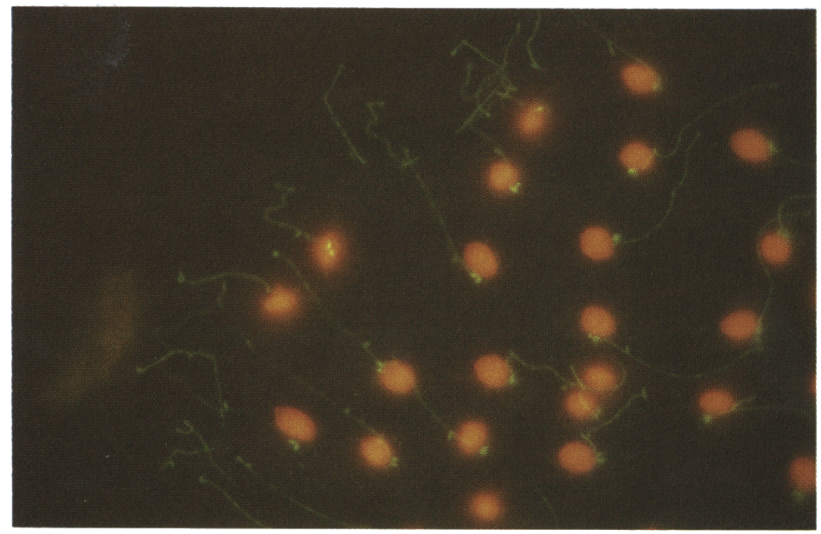

Figure 4. Staining of Spec3 and nuclei. Prism-stage embryos were immunofluorescently stained for Spec3 and counterstained with ethidium bromide to highlight nuclei. peptides, as well as genuine $\mathrm{KLH}$, on immunoblots. An antiserum raised against just $\mathrm{KLH}$ showed only weak and diffuse staining of embryos, similar to that observed for staining with preimmune serum or the secondary antibody alone (Figure 5F). Of several antisera raised against a variety of echinoid and foreign proteins (including tubulins), which we have used to stain embryos, only the Spec3 antiserum showed the distinctive pattern of staining of the cilia and Golgi apparati. These observations indicate that staining of the cilia and Golgi complex is not due to an epitope on KLH or adventitious binding. Figure 5 shows that preincubation of the stripped antiserum with the Spec3 amino-terminal peptide, or with the peptide conjugated to KLH used to raise the antiserum, prevented subsequent staining of the cilia (Fig. 5A,C,D), whereas a nonrelevant peptide conjugated to KLH (or KLH alone) did not block staining (Fig. 5B). Unaccountably, the Golgi complex continued to stain even when the concentration of blocking peptide was increased sevenfold (Fig. 5E). Collectively, these controls indicate the specificity of the Spec3 antiserum against the epitope(s) present on the Spec3 protein and on cilia. Below we present additional evidence that the same antigen is being recognized in both cilia and the Golgi apparati.
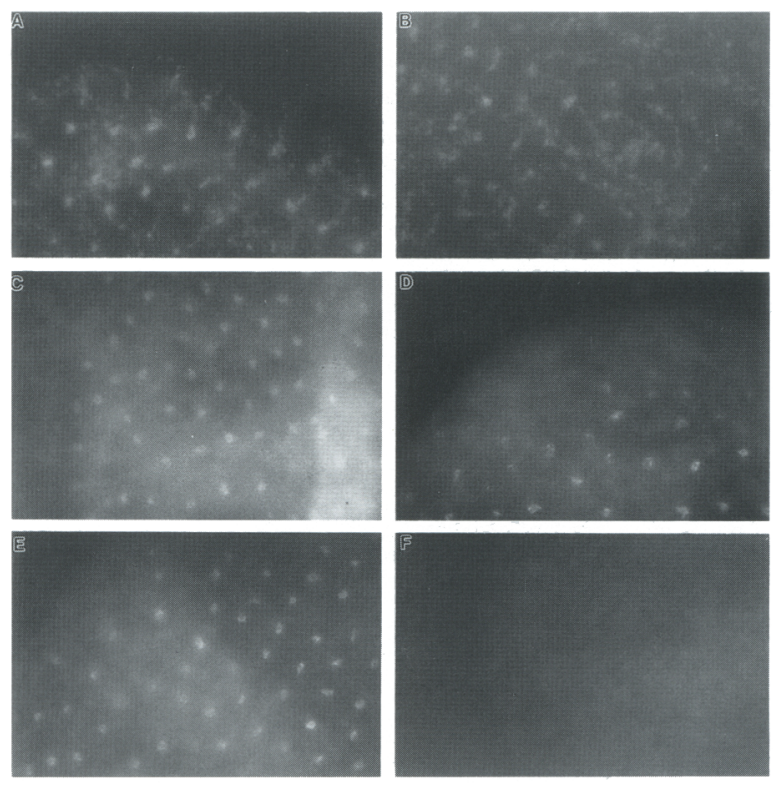

Figure 5. Specificity of Spec3 antibody staining. $(A-E)$ Immunofluorescent images of embryos at prism-stage labeled with Spec3 antisera at $1: 200$ dilution. (A) Antiserum was not preabsorbed. $(B)$ Antiserum was preabsorbed at $100 \mu \mathrm{g} / \mathrm{ml}$ with an unrelated synthetic peptide conjugated to KLH. (C) Antiserum was preabsorbed at $100 \mu \mathrm{g} / \mathrm{ml}$ with the amino-terminal Spec3 synthetic peptide conjugated to KLH. (D) Antiserum was preabsorbed at $100 \mu \mathrm{g} / \mathrm{ml}$ with Spec3 synthetic peptide alone. $(E)$ Antiserum was preabsorbed at higher concentration of $700 \mu \mathrm{g} / \mathrm{ml}$ with Spec3 synthetic peptide alone. Even at a dilution of $1: 40,000$ and preabsorption with Spec3 synthetic peptide at $700 \mu \mathrm{g} / \mathrm{ml}$, Spec3 antiserum stained Golgi complex weakly, but at a detectable level. $(F)$ Immunofluorescent image of embryo at prism-stage, labeled with KLH antiserum at $1: 200$ dilution. 


\section{Immunogold localization of the Spec3 protein}

We used the Spec3 antiserum, in conjunction with a secondary antiserum conjugated to colloidal gold, to label thin sections of pluteus-stage embryos. As represented in Figure 6A, gold particles were observed along the length of the cilia of ectodermal cells; cilia of endodermal cells from the same embryos were not labeled (Fig. 6B). Specifically labeled ectodermal cilia are shown at higher magnification in Figure 6F; few gold particles were observed along cilia treated with preimmune antisera (e.g., Fig. 6C). Much of the labeling appears to be on the surface of the cilia, suggesting that at least the amino-terminal portion of the Spec3 protein is on the surface of the cilia.

A clump of gold spheres just beneath the cilium in Figure 6A may indicate labeling of an internal structure. Clusters of colloidal gold associated with vesicular structures were frequently observed in the apical cytoplasm beneath the cilium. Although the labeled structures cannot always be identified, as in Figure 6A, the stacks of Golgi cisternae were clearly labeled in many
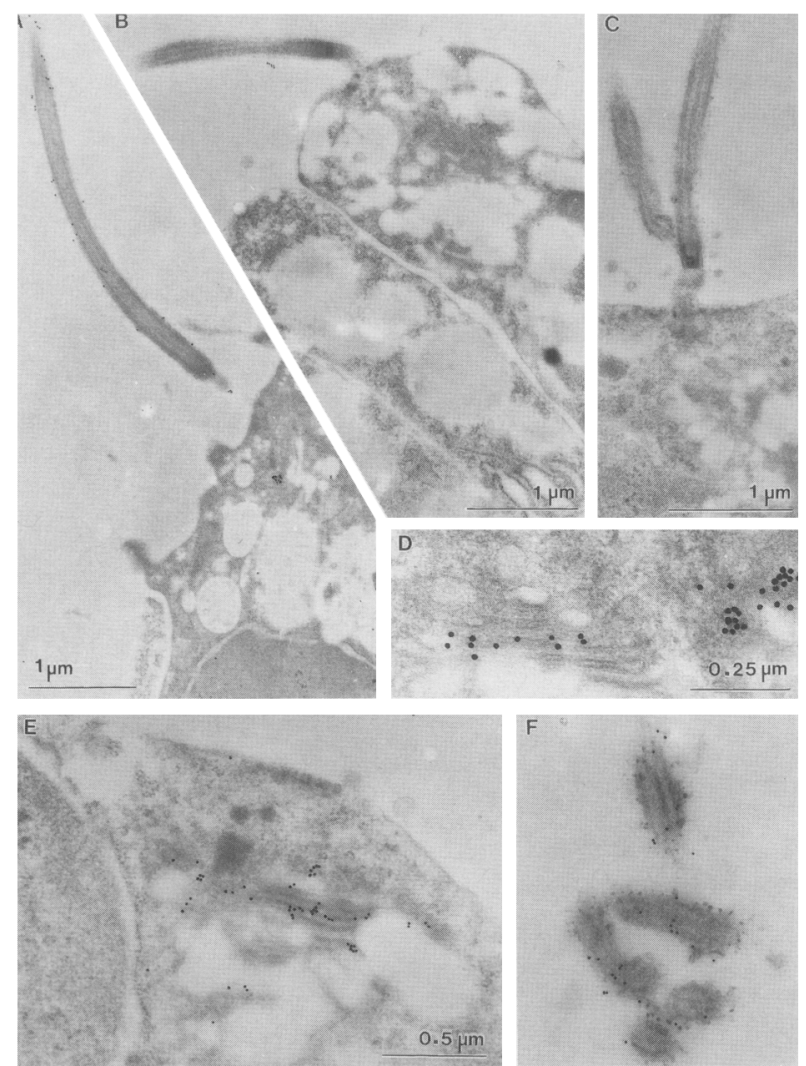

Figure 6. Immunogold staining of Spec3 with pluteus-stage embryos. Sections of embryos fixed in glutaraldehyde and paraformaldehyde were incubated with either Spec3 or preimmune antisera, followed by incubation with $10-\mathrm{nm}$ colloidal goldconjugated antibodies against rabbit IgGs. $(A)$ Ectoderm cell of a section exposed to Spec3 antiserum. (B) Ciliated endoderm cell exposed to Spec3 antiserum. $(C)$ Cilium exposed to preimmune antiserum. ( $D$ and $E$ ) Apical regions of ectoderm cells stained for Spec 3 and enlarged to show Golgi complex. $(F)$ Cilia in cross section exposed to Spec3 antiserum. cases (Fig. 6D and E). Though Golgi apparati were not always labeled, we never saw consistent immunogold labeling of other identifiable intracellular organelles. In particular, the basal bodies and ciliary rootlets were not labeled. The cilia of embryos treated with colcemid and examined by immunogold electron microscopy were labeled to the same degree as untreated controls, but the gold was dispersed throughout the cytoplasm rather than being concentrated at the apex (data not shown); this is consistent with the immunofluorescent staining of embryos treated with colcemid.

To better document the position of the Golgi apparati relative to the base of the cilium, embryos were fixed and thin-sectioned for conventional transmission electron microscopy. Figure 7 shows the apical regions of an ectodermal cell: The stacks of Golgi cisternae are closely apposed to the basal body and striated rootlets. This close association of Golgi cisternae with cilia is typical of that observed in other cells (Cavalier-Smith 1982). The dimensions of the Golgi apparati observed in thin sections were similar to the dimensions of the structure brightly stained by immunofluorescence.

\section{Profile of Spec3 staining during embryogenesis}

The characteristic pattern of staining of ectoderm cells reported above was observed for gastrula and later embryo stages. Golgi complexes and cilia of oral and aboral ectoderm cells were stained. Brightly stained apical structures or cilia were never observed in endoderm cells (which are ciliated) or mesenchyme cells, consistent with the great enrichment of Spec 3 mRNA in the ectoderm (Eldon et al. 1987).

Because Spec3 mRNA accumulates maximally just prior to hatching, we investigated the distribution of the Spec3 protein in blastulae. Just after hatching, when the cilia were actively beating, the pattern of staining was

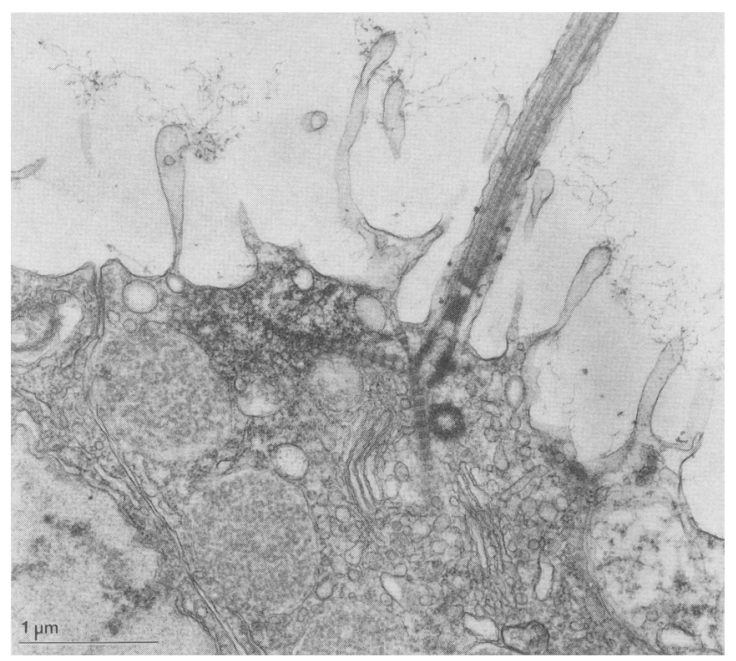

Figure 7. Transmission electron micrograph of Golgi complexes of ectoderm cells. The apical region of an ectoderm cell is shown for a prism-stage embryo. Fixation was in the presence of ruthenium red. 

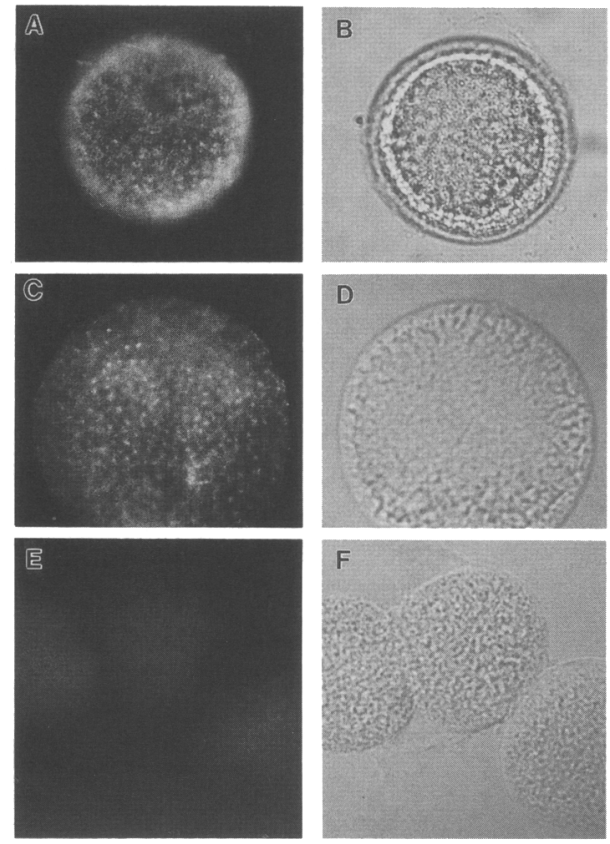

Figure 8. Blastula-stage embryos and unfertilized eggs labeled with Spec3 antibody. (A) Immunofluorescent image of hatched blastula labeled with Spec3 antiserum. $(B)$ Bright-field image of embryo in $A$. $(C)$ Immunofluorescent image of blastula labeled with Spec3 antiserum. $(D)$ Bright-field image of embryo in $C$. $|E|$ Immunofluorescent image of unfertilized eggs labeled with Spec3 antiserum. $(F)$ Bright-field image of eggs in $E . C$ and $E$ were stained, photographed, and printed under the same conditions to show relative labeling intensities.

quite different from that observed at later stages. The fluorescent stain was broadly distributed throughout the cytoplasm in a fine punctate pattern and not concentrated in the apical zone (Fig. 8A); staining of cilia was faint or undetectable. Ingressing mesenchyme and vegetal plate cells did not stain significantly. In some cells of the blastula shown in Figure 8C, Spec3 staining, although generally diffuse, showed discrete centers of higher concentration. It thus appears that in hatched blastulae the Spec3 protein is synthesized throughout the cytoplasm of ectodermal cells and gradually coalesces into the characteristic discrete pattern as it accumulates in Golgi apparati and on cilia at later stages.

Although the level of Spec3 mRNA was very low in the egg and early embryo, there may be a maternal store of Spec3 protein. Blastulae (Fig. 8C) and unfertilized eggs (Fig. 8E) were stained in parallel and photomicroscopy performed under the same exposure conditions. Spec3 antigen was present at low levels, if at all, in eggs but had increased considerably by blastula stage. The paucity of Spec3 mRNA and antigen in eggs and early embryos indicates that its functioning is not required until later embryogenesis.

When pluteus-stage embryos were immunofluorescently stained with Spec3 antiserum, the characteristic pattern of brightly stained Golgi complexes and associated cilia of ectodermal cells was observed, though the complexes appeared to be smaller than those in gas- trulae. At low resolution, a bright cloud of stain appeared at the intersection of the oral and aboral surfaces of the embryo. As shown in Figure 9, this cloud was attributable to the bright staining of the dense cluster of cilia of the small, closely spaced cells of the ciliated band. The cytoplasm of these cells stained intensely too, but the large, brightly stained Golgi complexes typical of other ectoderm cells were not observed.

\section{Structural characteristics of the Spec3 protein}

Protein extracts of prism-stage embryos of $S$. purpuratus were separated by SDS-PAGE, blotted onto Immobilon-P membranes, and stained with Spec3 antiserum. As shown in Figure 10A, nearly all of the staining corresponded to a diffuse band (sometimes resolved into two bands) that barely entered the gel, even at polyacrylamide concentrations of only $5 \%$. Comparison with the mobility of the more rapidly migrating dynein heavy chains prepared from isolated cilia (Bell et al. 1982) indicated that the stained material has an $M_{\mathrm{r}}>350,000$, far greater than the predicted Spec3 $M_{\mathrm{r}}$ of 21,600 , based on the open reading frame of the Spec3 cDNA. Such a discrepancy in apparent molecular weight cannot be accounted for by conventional post-translational modifications. The mobility was unaltered by alkylation of the reduced extract before electrophoresis (Fig. 10C) or preparation of samples at low temperature. It is likely that the slowly migrating band represents a complex that includes the Spec3 antigen. The stained band was greatly enriched in a preparation of cilia (Fig. 10A, lane 1) compared with extracts of whole embryos (Fig. 10A, lane 3), when approximately equal amounts of protein were loaded on each lane. The stained band was observed in extracts of cilia prepared from prism- (as well as mesenchyme blastula- and gastrula-) stage embryos, but was greatly reduced in cilia extracts from hatching blastulae (Fig. 10C), consistent with the appearance of the Spec3

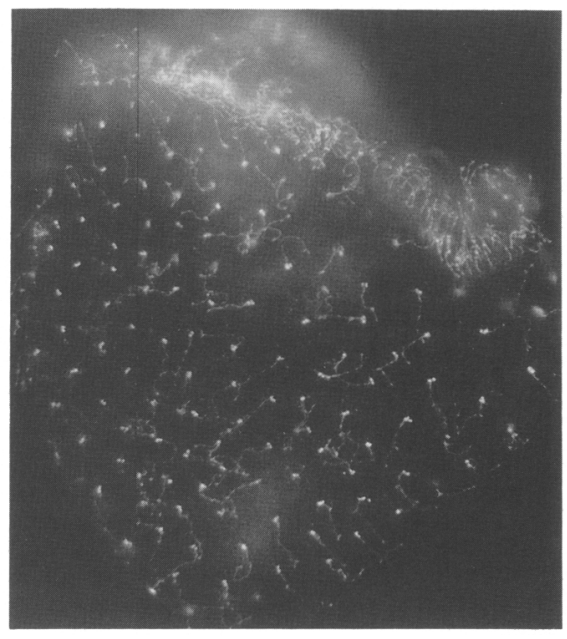

Figure 9. Pluteus-stage embryo labeled with Spec3 antiserum. Immunofluorescent image of an embryo stained with Spec3 antiserum. A composite of two micrographs of the same embryo is shown. 


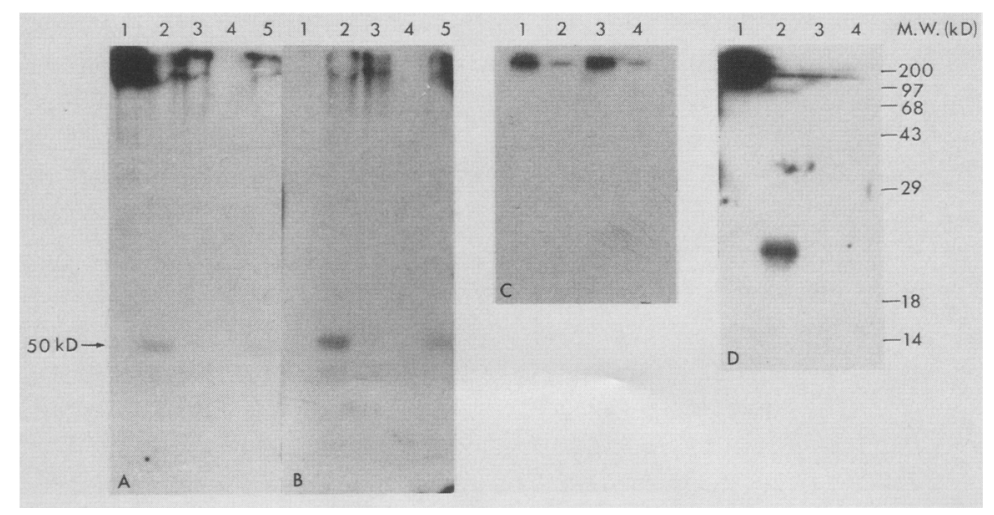

Figure 10. Characterization of the Spec3 protein. $(A)$ An immunoblot stained with the Spec3 antiserum of proteins solubilized from isolated prism-stage cilia (lane 1), deciliated (lane 2) or intact prism-stage embryos (lane 3), unfertilized eggs (lane 4), or deciliated prism-stage embryos after ciliary regeneration (lane 5). (B) An immunoblot prepared in parallel with $A$ stained with Spec3 antiserum preincubated with the Spec3 amino-terminal peptide was used to raise the antiserum. (C) An immunoblot of cilia proteins prepared from prism-stage (lanes 1 and 3) and hatching blastulastage (lanes 2 and 4) emiryos stained with Spec3 antiserum. Proteins shown in lanes 3 and 4 were reduced and alkylated before electrophoresis. Equal amounts of protein were loaded onto each lane. $(D)$ An immuno-

blot stained with Spec3 antiserum showing proteins prepared from cilia of $S$. purpuratus (lane 1) and L. pictus prism-stage embryos (lane 2), or intact $L$. pictus prisms (lane 3) or eggs (lane 4). $(A, B$, and $D)$ Each lane was loaded with samples corresponding to equal amounts of protein.

antigen on stained cilia after hatching. Furthermore, unfertilized eggs showed no reaction (Fig. 10A, lane 4). Deciliation of embryos resulted in a decline in the staining of the band (Fig. 10A, lane 2), which was restored after regeneration of cilia. Deciliation also resulted in the transient appearance of a faintly staining band with an $M_{\mathrm{r}} \sim 50,000$, which disappeared after about $2 \mathrm{hr}$ of regeneration (Fig. 10A, lanes 2 and 5). Because deciliation results in a transient increase in the level of Spec3 mRNA (Eldon et al. 1987), the 50,000-dalton form might be a precursor in the formation of the large complex that stains for Spec3.

Preincubation of the Spec3 antiserum with the Spec3 amino-terminal peptide used for immunization almost completely blocked the intense staining of the ciliary protein (Fig. 10B, lane 1). On the other hand, the much fainter signal observed for extracts of whole, regenerated, or deciliated embryos was only slightly reduced by the blocking peptide. These results are similar to the blocking of the immunocytochemical staining of cilia but not the Golgi complex. An interpretation is that the Spec3 antiserum reacts with a ciliary antigen that can be fully blocked and with a Golgi antigen that is indistinguishable by electrophoretic mobility but is altered in some way such that its staining cannot be blocked effectively. It is thus likely that these antigens are closely related or identical, but we cannot exclude the possibility that they simply share an epitope and electrophoretic properties. The staining of the 50,000-dalton band that appeared after deciliation could not be blocked either, extending the likelihood of a relationship between the Spec3 antigens of cytoplasmic extracts and cilia.

\section{The Spec3 antigen in L. pictus}

Recently, we isolated a Spec3 cDNA homolog from a distantly related sea urchin, $L$. pictus. Its sequence analysis shows considerable similarity of the two proteins ( $88 \%$ amino acid sequence identity), implying that the Spec3 protein has a conserved function in echinoid embryos (Z. Gong, T. Nguyen, B.P. Brandhorst, and W.H. Klein, unpubl.). In spite of a $64 \%$ identity in the deduced amino acid sequence between the peptide used to gen- erate the antiserum for these investigations and the amino terminus of the L. pictus Spec3 homolog, there was very weak or no detectable immunostaining of $L$. pictus embryos with the Spec 3 antiserum even with very low dilutions of antiserum.

However, immunoblots of $L$. pictus proteins extracted from unfertilized eggs, prism-stage embryos, or cilia isolated from prism-stage embryos yielded an unexpected result. The Spec3 antigen was greatly enriched in the cilia of $L$. pictus, as observed for S. purpuratus (Fig. 10D, lane 2 versus lane 3 or 4). However, in this case, the antigen migrated as a diffuse band at $M_{r} \sim 23,000$, rather than the huge aggregate observed with $S$. purpuratus cilia (Fig. 10D, lane 1 versus lane 2). The intensity of the cross-reacting material in $L$. pictus was much weaker than that in $S$. purpuratus, consistent with the lack of signal observed when embryos were stained for immunofluorescence. These results indicate that the Spec3 antiserum indeed recognizes a ciliary Spec3 protein, as defined by its open reading frame, and suggest that the Spec3 complex is more readily disaggregated to its monomer form in L. pictus than in S. purpuratus.

\section{Localization of the Spec3 antigen on cilia}

The immunogold staining of cilia suggested that the Spec3 antiserum used recognizes an antigen that resides, at least in part, on the surface of the cilium. As shown in Figure 11, brief treatment of prism-stage embryos with trypsin changed the characteristic beaded staining pattern to a uniform one. The pattern and intensity was not obviously altered further when the 1 -min treatment was extended to $10 \mathrm{~min}$. The cilia of embryos treated with trypsin continued to beat actively, and the cells excluded trypan blue. Thus, trypsin treatment must have disrupted something on the cilium surface.

The deduced amino acid sequence indicates that the Spec3 protein should be cleaved by trypsin at five sites. Because the intensity of staining of the cilium decreased little, if at all, during the initial minute of digestion and no more after $10 \mathrm{~min}$, it is unlikely that a Spec3 aminoterminal peptide was cleaved by trypsin and released. The change from beaded to uniform staining pattern 

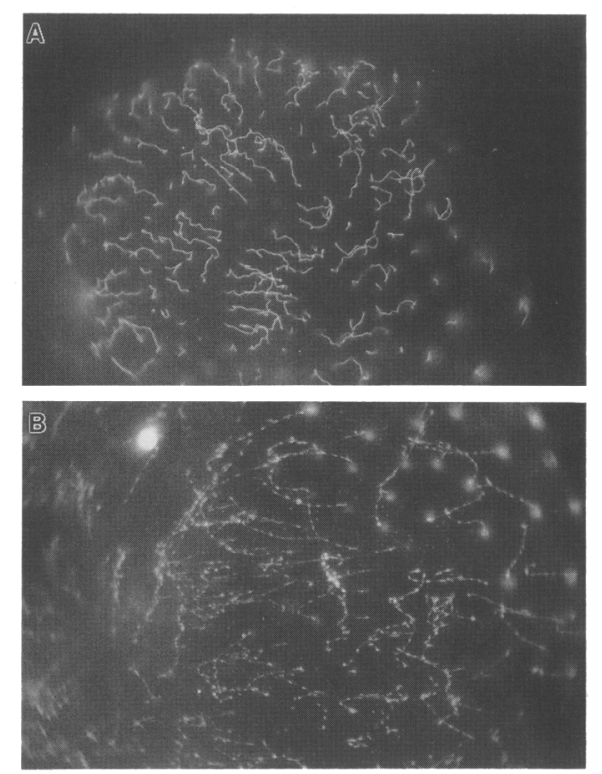

Figure 11. Alteration of Spec3 staining pattern on cilia by trypsin treatment. Prism-stage embryos were treated with 250 $\mu \mathrm{g} / \mathrm{ml}$ trypsin for $1 \mathrm{~min}$, fixed in methanol, and stained for Spec3. (A) Field of cilia of a treated embryo; $(B)$ field of cilia of an untreated embryo prepared at the same time and photographed under the same exposure conditions as $A$.

might result from increased exposure of Spec3 antigenic sites normally protected from reaction with the antiserum. This explanation is consistent with the presence of the Spec3 antigen on the outer surface of the ciliary membrane, but it is possible that a trypsin-induced alteration in surface topology of the cilium is transduced through the plasma membrane to internal structural elements with which Spec3 might be associated.

If Spec3 is a membrane protein, it should fractionate with ciliary membranes. A whole membrane fraction was prepared from cilia by extraction with Triton X-100 (Stephens 1977), whereas integral and other membrane proteins were separated by differential extraction with Triton X-114 (Dentler 1988). As shown in Figure 12, the majority of the Spec 3 antigen remained associated with the axonemal residual pellets, indicating that it is not strictly a membrane protein. These pellets were extracted with a high salt buffer under conditions that solubilize dyneins of sperm flagellar axonemes (Bell et al. 19821, or half the dyneins and all of the B-subfibers of ciliary axonemes (Stephens 1986). It is clear that the Spec3 antigen is closely associated with the residual axoneme.

\section{Discussion}

Although a function for the Spec3 protein cannot yet be proposed, we have localized the Spec3 amino-terminal antigen to the cilia and have shown that it migrates as a large aggregate on SDS-PAGE. Moreover, our results indicate that the strikingly ordered particulate staining pattern of ectodermal cells is attributable to the staining of the Golgi complex with the Spec3 antiserum. This staining is due to a cytoplasmic antigen having electrophoretic properties indistinguishable from those of the ciliary Spec3 antigen.

Interpretation of our results is complicated by the failure of the Spec3 amino-terminal peptide to block the staining of the Golgi complex or the cytoplasmic form of the Spec3 antigen detected on immunoblots of deciliated embryos. However, the immunological cross-reactivity of the ciliary and cytoplasmic antigens, their indistinguishable electrophoretic properties, and their similar ontogenetic patterns of accumulation indicate a strong similarity, if not identity, of the two localized antigens and their relatedness to the Spec3 protein. The failure of the blocking peptide to compete with the Golgi antigen is not simply due to adventitious staining of the Golgi complex, because staining was never observed with preimmune serum or other rabbit antisera raised against a variety of antigens, including the KLH used to conjugate the Spec3 peptide for generation of the antiserum.

We cannot interpret the behavior of the $S$. purpuratus Spec3 antigen on SDS-PAGE, which normally dissociates noncovalent complexes of proteins. The failure of a reducing and blocking step to reduce the mobility indicates that the apparent complex with which Spec3 is associated is not based on cystine cross-links. The Spec3 protein probably interacts with itself or other proteins to form an aggregate that is very resistant to dissociation. Unexpectedly, the electrophoretic properties of Spec3 extracted from $L$. pictus cilia were different. In this case, the protein migrated with an apparent molecular weight close to that predicted from the sequence of the Spec3 cDNA clone. We do not understand the basis for this difference, though it seems reasonable to hypothesize that Spec3 exists in a similar molecular environment in both species but is more readily disaggregated in $L$.

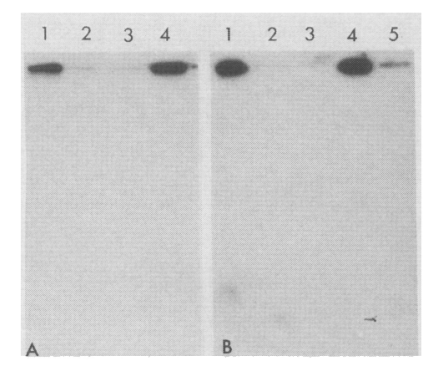

Figure 12. Immunoblot analysis of membrane and axonemal fractions of cilia using two different methods of fractionation of ciliary membrane proteins. (A) Membrane proteins were solubilized by extraction of isolated cilia with Triton X-100. $(B)$ Cilia were differentially extracted with Triton X-114 to yield integral (lane 3) and other (lane 2) membrane proteins. In both instances, the demembranated axonemal pellets were extracted with a high salt buffer to solubilize dyneins and B-subfiber proteins $(A$, lane $3 ; B$, lane 5$)$; the proteins of the residual pellets are shown in lane 4 . Lane 1 show proteins solubilized from whole cilia. Samples corresponding to equal amounts of cilia were loaded onto each lane. 
pictus. This could be due to differences in the sequence between the two proteins or to differences in proteins that may interact with Spec3. Further evidence for the capacity of the Spec3 protein to aggregate was provided by analysis of the products of translation of synthetic Spec3 mRNA in a rabbit reticulocyte lysate. The radiolabeled products showed a complex pattern on SDSPAGE, most migrating considerably more slowly than expected for the Spec3 protein, though the products migrated faster than the Spec3 antigen extracted from embryos (Eldon 1989). Preliminary experiments suggested that these translation products, including some material at 23,000 daltons, could be immunoprecipitated with the Spec3 antiserum (E.D. Eldon and W.H. Klein, unpubl.). The Spec3 protein thus appears to be capable of forming highly stable aggregates with itself and/or a variety of other echinoid and foreign proteins. The physical properties of the Spec3 antigen, like the amino acid sequence of Spec3, thus appear to be unusual.

Previous measurements of the accumulation of Spec3 mRNA during embryogenesis (Eldon et al. 1987) can be compared with the immunochemical data. Spec3 transcripts are present at low levels in unfertilized eggs but accumulate rapidly during cleavage, increasing 50-fold by hatching blastula stage. Transcript levels then decline abruptly, remain low during mesenchyme blastula and gastrula stages, and increase again during prism and pluteus stages. Immunofluorescent staining of unfertilized blastula, gastrula, prism, and plutei revealed where and when the Spec3 protein was present. Staining and immunoblots indicated very low levels (if any) of Spec3 protein in unfertilized eggs, consistent with the low prevalence of Spec3 mRNA. The cytoplasm of ectoderm cells of the blastula was intensely stained. The diffuse staining pattern seen in cells of hatched blastulae seemed to coalesce into the particulate pattern seen from the gastrula through pluteus stages. A simple model is that in early blastula stage embryos, when Spec 3 mRNA levels are high, the protein is being rapidly synthesized, presumably on the rough endoplasmic reticulum. The newly synthesized Spec3 protein then coalesces into the discrete particulate pattern as it is transported to, and accumulates in, the Golgi complex. There, the level of Spec3 protein increases as the rate of its synthesis decreases. The continued presence of high levels of Spec3 protein in the Golgi after the initial round of ciliogenesis suggests that post-translational processing and/or transport out of the Golgi is a slow step and that Spec3 is turned over on the cilium, a phenomenon reported by Stephens (1977) for protein components of the ciliary membrane. Treatment of embryos with monensin would inhibit transport of the Spec3 protein to the cilium, resulting in its depletion.

The intense immunofluorescent labeling of the ciliary band region of early pluteus stage embryos is consistent with in situ hybridization experiments using a Spec3 probe (Eldon et al. 1987). These experiments showed high levels of Spec3 mRNA in the ciliated band region. The formation of the ciliated band, a distinct cluster of ciliated columnar cells between the oral and aboral sur- faces, coincides with a rise in Spec3 message levels. From the in situ hybridization analysis, it appears that the increase in message takes place solely in the cells of the ciliated band. Thus, the high levels of Spec3 message and protein in this region presumably represent a second round of synthesis of the Spec3 protein prior to its transport to the Golgi and cilia. The absence of intensely stained Golgi complexes in these cells suggests that the Spec3 protein is more rapidly transported to the cilium.

The model suggests that the Spec3 protein may be targeted via the Golgi complex to the ciliary membrane or surface in a manner similar to the targeting of many membrane or secretory proteins (Garoff 1985). The presence of a Spec3 antigen in the Golgi complex, the disappearance of the Spec 3 antigen from the cilia after monensin treatment, the predominant localization of the Spec3 antigen to the surface of cilia by immunogold staining, and the alteration in staining pattern of cilia by mild trypsin treatment are all consistent with the Spec3 protein being a membrane or secreted surface protein. However, such evidence is not compelling. If the Spec3 protein indeed resides on the ciliary surface, it might be expected to have a signal peptide cleaved during insertion into the rough endoplasmic reticulum, as is characteristic of many secreted and trans-membrane proteins (von Heijne 1981). However, we can conclude that the Spec3 protein has no cleaved signal peptide. The deduced sequence of the amino terminus of the protein does not have the three-part structure typical of cleaved signal sequences (von Heijne 1981, 1985). Furthermore, the antisera used in the studies reported here were raised against the amino-terminal 14 amino acids, all of which would have been lost if the typical 15- to 25-residue signal sequence were cleaved. Some secreted proteins, such as ovalbumin (Braell and Lodish 1982; Meek et al. 1982), employ signal sequences near the amino terminus that are not removed. Similarly, several proteins have been identified that can be cotranslationally translocated across the endoplasmic reticulum without a cleaved signal peptide (Garoff 1985), having internal hydrophobic residues that serve as signal peptides. Many secreted and cell-surface proteins are glycosylated during their post-translational processing; the Spec3 protein has a single potential site for amino-linked glycosylation (Fig. 1; Eldon et al. 1987).

Evidence contradicting the hypothesis that Spec3 is a ciliary surface or membrane protein is that the Spec3 antigen fails to be extracted with nonionic detergents or to fractionate with the integral membrane protein fraction of cilia. It is possible, though, that the Spec3 antigen is part of a complex that is both tightly associated with the axoneme and penetrates the ciliary membrane. The data do not exclude the possibility that the Spec3 antigen associated with the Golgi apparatus is an integral part of the secretory complex and that another form of Spec3 antigen is directed to the cilia via a different pathway. This seems unlikely in view of the observation that when embryos are treated with monensin, which prevents secretion of proteins from the Golgi, detectable Spec3 antigen is lost from the cilia within $2.5 \mathrm{hr}$. 
Because the Spec3 antigen appears only on ectodermal cilia and only after cilia begin to beat, it is not an essential structural component of cilia, and its function is not required for beating. The Spec3 protein may be involved in the interactions of the embryo with its environment. For instance, in Chlamydomonas, proteins on the flagellar surface mediate the interaction of different mating types (Goodenough et al. 1980). Alternatively, Spec3 could be associated with the control of ciliary motion, which may change during embryogenesis in preparation for feeding or avoidance behavior. The actual function of Spec 3 can only be established by abolishing its activity and observing a specific defect. A potential method for accomplishing this would be to eliminate Spec3 expression by use of antisense RNA and look for defects in swimming behavior. The Spec3 antiserum does not stain flagellae of sperm of $S$. purpuratus or immunoblots thereof, indicating that it is a specialized ciliary protein. It remains to be established whether the presence and function of Spec3 protein is restricted to echinoid embryos.

\section{Methods}

\section{Embryo cultures}

$S$. purpuratus and $L$. pictus were obtained from Marinus, Inc., and $S$. purpuratus embryos were cultured at $16^{\circ} \mathrm{C}$, as described previously (Klein et al. 1987). L. pictus was cultured at $18^{\circ} \mathrm{C}$. Hatched blastula-, gastrula-, prism-, and pluteus-stage embryos occur $20,48,72$, and $96 \mathrm{hr}$ after fertilization, respectively, under our culture conditions for $S$. purpuratus. For L. pictus, prism-stage is reached at $48 \mathrm{hr}$ after fertilization. Embryos were deciliated as described in Eldon et al. (1987) Cilia were prepared according to Eldon (1989).

\section{Production of antiserum directed against a synthetic peptide}

A peptide corresponding to amino acids $1-14$ with an additional cysteine residue to provide a free sulfhydryl group was synthesized by Dr. Ralph Arlinghaus, (Department of Molecular Pathology, M.D. Anderson Cancer Center). It was coupled to $\mathrm{KLH}$ through a disulfide bond activated by treating the hemocyanin with $m$-maleimidobenzoyl- $N$-hydroxysuccinimide ester (MBS), following the protocol of Liu et al. (1979). Two young male New Zealand white rabbits were injected with a conjugate containing $\sim 140 \mu \mathrm{g}$ of hemocyanin and $100 \mu \mathrm{g}$ of peptide, and one was injected with $200 \mu \mathrm{g}$ of hemocyanin.

In all cases, antigen was resuspended in $\sim 1 \mathrm{ml}$ of sterile phosphate-buffered saline (PBS) and emulsified with either $1 \mathrm{ml}$ of complete Freund's adjuvant (for the first injection) or $1 \mathrm{ml}$ of incomplete Freund's adjuvant (for all subsequent injections). Animals were injected three times at 2 -week intervals prior to their first immune bleeding 10 days after the third injection. Blood was obtained through the marginal ear vein. Additional sera were obtained by injecting the animals and bleeding them 10 days later. Blood was collected in sterile tubes and incubated at $37^{\circ} \mathrm{C}$ for $1-2 \mathrm{hr}$ to ensure good clotting and then incubated at $4^{\circ} \mathrm{C}$ for $8-12 \mathrm{hr}$ to allow the clot to contract. Serum was decanted from the contracted clot and clarified by spinning for 30 min in a clinical centrifuge at $4^{\circ} \mathrm{C}$. The clarified sera were aliquoted into sterile tubes and stored at $-80^{\circ} \mathrm{C}$.

An IgG fraction was isolated by passing dialyzed serum over a DEAE Affi-Gel Blue column (Bio-Rad Laboratories). Antibodies directed against KLH were removed by affinity chromatog- raphy. The IgG fraction was passed over a Sepharose $4 \mathrm{~B}$ column (Pharmacia) to which KLH was bound (courtesy of Dr. Harry Woodward). Results of an ELISA (data not shown) demonstrated that the stripped IgG fraction retains most of its antipeptide titer but loses most of its antihemocyanin titer; this stripped preparation was used in all investigations reported.

\section{Fluorescent labeling of whole embryos}

Whole embryos of $S$. purpuratus were fixed for $6 \mathrm{~min}$ in $90 \%$ methanol, $10 \mathrm{mM}$ EDTA (or EGTA) at $-20^{\circ} \mathrm{C}$, following the protocol of Harris (1986). Embryos were either settled onto polylysine-coated coverslips or treated by batch in $1.5-\mathrm{ml}$ microcentrifuge tubes. Following rehydration, embryos were blocked with phosphate-buffered saline (PBS) containing $10 \%$ fetal calf serum and $3 \%$ bovine serum albumin (PBS/FCS/BSA) for at least $1 \mathrm{hr}$ at either $4^{\circ} \mathrm{C}$ or room temperature. The blocking buffer was replaced with IgGs at a concentration of $50 \mu \mathrm{g} / \mathrm{ml}$ in $\mathrm{PBS} / \mathrm{FCS} / \mathrm{BSA}$ and incubated for at least $1 \mathrm{hr}$. In some instances, prior to replacing the blocking buffer, IgGs at $1: 200$ dilution in PBS/FCS/BSA were preabsorbed for $30 \mathrm{~min}$ at room temperature with the amino-terminal Spec3 peptide or with the peptide conjugated to $\mathrm{KLH}$. The samples were washed three times for at least $10 \mathrm{~min}$ each in PBS. The fluorescein-conjugated antirabbit IgGs (no. 1612-3151, Cooper Biomedical) were diluted $1: 30$ in PBS/FCS/BSA, applied to the samples, and incubated for at least $1 \mathrm{hr}$. Following three more washes in PBS, samples were mounted under a coverslip in a drop of $1 \mathrm{mg} / \mathrm{ml}$ paraphenylenediamine (to retard bleaching; Valnes and Brandtzaeg 1985) in PBS, and examined and photographed with a Zeiss IM35 microscope. Nuclei were sometimes counterstained with $0.0001 \%$ ethidium bromide in PBS just prior to viewing.

\section{Preparation of sectioned samples for electron microscopy}

Samples were prepared for immunoelectron microscopy following previously described protocols (Decker et al. 1988). Briefly, thin sections of embryos embedded in Lowicryl K4M were exposed for $60 \mathrm{~min}$ to $20 \mu \mathrm{g} / \mathrm{ml}$ of the primary antibody directed at Spec3. Subsequently, following a brief wash in buffer, anti-rabbit IgG coupled to colloidal gold (Janssen Life Sciences Products, Olen, Belgium/ was diluted one-tenth and similarly applied to the sections. After being poststained with $2 \%$ aqueous uranyl acetate, the specimens were photographed in a Phillips 410 electron microscope operated at $60 \mathrm{kV}$. Specimens prepared for conventional electron microscopy were fixed in a mixture of glutaraldehyde, osmium tetroxide, and ruthenium red (Galileo and Morrill 1985) and subsequently dehydrated and embedded in Spurr resin, as described previously (Decker and Lennarz 1979).

\section{Immunoblot analysis of sea urchin embryo extracts}

Protein extract from $S$. purpuratus or L. pictus embryos was run on $7.5 \%$ SDS-PAGE and electrotransferred as described in Towbin et al. (1979), with the exception that $0.1 \%$ SDS was present in the electrode buffer. Also, Immobilon-P transfer membranes (Millipore) were used in place of nitrocellulose membranes. The transferred Immobilon-P filters were probed with rabbit antisera and incubated with ${ }^{125}$ I-labeled donkey anti-rabbit secondary antibodies or protein $\mathrm{A}$ and followed by autoradiography.

\section{Fractionation of ciliary proteins}

Cilia were prepared from embryos deciliated by hypertonic shock, as described by Stephens (1986). Membrane proteins 
were solubilized from isolated cilia by extraction with Triton X-100, as described by Stephens (1977). Integral membrane proteins were separated from other membrane proteins by differential solubilization in Triton X-114 (Dentler 1988). The residual axonemal pellets were extracted with a high salt buffer $(0.6 \mathrm{M} \mathrm{NaCl})$, according to Bell et al. (1982), which releases dyneins. The proteins of each soluble fraction were precipitated in $80 \%$ acetone, and all fractions including the residual pellet were dissolved in SDS-sample buffer, separated on $10 \%$ SDSPAGE, and blotted onto Immobilon-P. The Spec3 protein was detected by reaction with $S p e c 3$ antiserum and iodinated protein A.

\section{Reduction and alkylation of proteins}

Proteins of isolated cilia were reduced and blocked, as described by Harlow and Lane (1988). Isolated cilia were diluted with water to a concentration of $1 \mathrm{mg} / \mathrm{ml}$. An equal volume of solution containing $1.5 \%$ SDS, $20 \mathrm{~mm}$ dithiothreitol, was added, and the mixture was incubated at $85^{\circ} \mathrm{C}$ for $10 \mathrm{~min}$. Fresh $N$-ethylmaleimide was added to a final concentration of $0.04 \mathrm{M}$, followed by incubation on ice for $1 \mathrm{hr}$. The sample was diluted with one-fifth volume of solution containing $50 \%$ glycerol, $0.5 \% \beta$-mercaptoethanol, and $0.001 \%$ bromphenol blue and analyzed on $10 \%$ SDS-PAGE.

\section{Acknowledgments}

We thank Dr. Robert Levine for advice and use of his microscope, Dr. Dan Carson for advice on the use of monensin, Sharon Bickel for advice on blotting, and Dr. Peter Gergen for use of his objectives. We thank Jeff Arnum and Guy L'Heureux for help with the figures. This work was supported by an National Institutes of Health grant (HD-22619) to W.H.K and NIH (HD-18322) and NSERC grants to B.P.B.

\section{References}

Allan, V.J. and T.E. Kreis. 1986. A microtubule-binding protein associated with membranes of the Golgi apparatus. J. Cell Biol. 103: 2229-2239.

Bell, C.W., C. Fraser, W.S. Sale, W.Y. Tang, and I.R. Gibbons. 1982. Preparation and purification of dynein. Methods Cell Biol. 24: 373-397.

Braell, W.A. and H.F. Hodish. 1982. Ovalbumin utilizes an $\mathrm{NH}_{2}$-terminal signal sequence. J. Biol. Chem. 257: 45784582.

Burke, R.D. 1978. The structure of the nervous system of the pluteus larva of Strongylocentrotus purpuratus. Cell Tissue Res. 191: 233-247.

1983. Development of the larval nervous system of the sand dollar, Dendraster excentricus. Cell Tissue Res. 229: $145-154$.

Cavalier-Smith, T. 1982. The evolutionary origin and phylogeny of eukaryotic flagella. Symp. Soc. Exp. Biol. 35: 465493.

Decker, G.L. and W.J. Lennarz. 1979. Sperm binding and formation of the fertilization envelope by surface complexes isolated from sea urchin eggs. J. Cell Biol. 81: 92-103.

Decker, G.L., M.C. Valdizan, G.M. Wessel, and W.J. Lennarz. 1988. Developmental distribution of a cell surface glycoprotein in the sea urchin Stronglyocentrotus purpuratus. Dev. Biol. 129: 339-349.

Dentler, W.L. 1988. Fractionation of Tetrahymena ciliary membranes with Triton X-114 and the identification of a ciliary membrane ATPase. J. Cell Biol. 107: 2679-2688.
Eldon, E.D. 1989. 'The Spec3 gene of Strongylocentrotus purpuratus encodes a component specific for embryonic ectodermal cilia'. Ph.D. thesis, Indiana University, Bloomington, Indiana.

Eldon, E.D., L.M. Angerer, R.C. Angerer, and W.H. Klein. 1987. Spec3: Embryonic expression of a sea urchin gene whose product is involved in ectodermal ciliogenesis. Genes Dev. 1: $1280-1292$.

Galileo, D.S. and J.B. Morrill. 1985. Patterns of cells and extracellular material of the sea urchin Lytechinus variegatus (Echinodermata; Echinodea) embryo, from hatched blastula to last gastrula. J. Morphol. 185: 387-402.

Garoff, H. 1985. Using recombinant DNA techniques to study protein targeting in the eukaryotic cell. Annu. Rev. Cell Biol. 1: 403-445.

Goodenough, U.W., W.S. Adair, E. Caligor, C.L. Forest, J.L. Hoffman, D.A.M. Meseand, and S. Spath. 1980. Membranemembrane and membrane-ligand interactions in Chalamydomas mating. In Membrane-membrane interactions, led. N.B. Gilula), pp. 131-152. Raven Press, New York.

Gong, Z. and B.P. Brandhorst. 1987. Stimulation of tubulin gene transcription by deciliation of sea urchin embryos. Mol. Cell. Biol. 7: 4238-4246.

Harlow, E. and D. Lane. 1988. Alkylation of proteins prior to running on a gel. In Antibodies: A laboratory manual, pp. 646, Cold Spring Harbor Laboratory, Cold Spring Harbor, New York.

Harlow, P. and M. Nemer. 1987. Developmental and tissue-specific regulation of $\beta$-tubulin gene expression in the embryo of the sea urchin Strongylocentrotus purpuratus. Genes Dev. 1: 147-160.

Harris, P.M. 1986. Cytology and immunocytochemistry. Methods Cell Biol. 27: 243-262.

Keller, L.R., J.A. Schloss, C.D. Silflow, and J.L. Rosenbaum. 1984. Transcription of $\alpha$ - and $\beta$-tubulin genes in vitro in isolated Chlamydomonas reinhardtii nuclei. J. Cell Biol. 98: $1138-1143$.

Klein, W.H., S.H. Hardin, and B.P. Brandhorst. 1987. Spec proteins: Calcium-binding proteins in the embryonic ectoderm cells of sea urchins. Methods Enzymol. 139: 354-363.

Lin, J.J.-C. and S.A. Queally. 1982. A monoclonal antibody that recognizes Golgi-associated protein of cultured fibroblast cells. J. Cell Biol. 92: 108-112.

Linck, R.W. and R.E. Stephens. 1987. Biochemical characterization of tektins from sperm flagellar doublet microtubules. $I$. Cell Biol. 104: 1069-1075.

Lipsky, N.G. and R.E. Pagano. 1985. A vital stain for the Golgi apparatus. Science 228: $745-747$.

Liu, F.-T., M. Zinnecker, T. Hamaoka, and D.H. Katz. 1979. New procedures for preparation and isolation of conjugates of proteins and a synthetic copolymer of D-amino acids and immunochemical characterization of such conjugates. Biochemistry 18: 690-697.

Luck, D.J.L., B. Huang, and G. Piperno. 1982. Genetic and biochemical analysis of the eukaryotic flagellum. Symp. Soc. Exp. Biol. 35: 399-419.

Lucocq, J.M. and G. Warren. 1987. Fragmentation and partitioning of the Golgi apparatus during mitosis in HeLa cells. EMBO J. 6: 3239-3246.

Masuda, M. 1979. Species-specific pattern of ciliogenesis in developing sea urchin embryos. Dev. Growth Differ. 21: $545-$ 552.

Masuda, M. and H. Sato. 1984. Asychronization of cell division is concurrently related with ciliogenesis in sea urchin blastulae. Dev. Growth Differ. 26: 281-294.

Meek, R.L., K.A. Walsh, and R.D. Palmiter. 1982. The signal 
sequence of ovalbumin is located near the $\mathrm{NH}_{2}$ terminus. $J$. Biol. Chem. 257: 12245-12251.

Miller, S.C., G.K. Pavlath, B.T. Blakely, and H.M. Blau. 1988. Muscle cell components dictate hepatocyte gene expression and the distribution of the Golgi apparatus in heterokaryons. Genes Dev. 2: 330-340.

Nakajima, Y. 1986a. Presence of a ciliary patch in preoral epithelium of sea urchin plutei. Dev. Growth Differ. 28: 243249.

1986b. Development of the nervous system of sea urchin embryos: Formation of ciliary bands and the appearance of two types of ectoneural cells in the pluteus. Dev. Growth Differ. 28: 531-542.

Ogawa, K. and I.R. Gibbons. 1976. Dynein 2: A new adenosine triphosphatase from sea urchin sperm flagella. J. Biol. Chem. 251: 5793-5801.

Raff, R.A. 1975. Regulation of microtubule synthesis and utilization during early embryonic development of the sea urchin. Am. Zool. 15: 661-678.

. 1977. Major membrane protein differences in cilia and flagella: Evidence for a membrane associated tubulin. Biochemistry. 16: 2047-2058.

1986. Isolation of embryonic cilia and sperm flagella. Methods Cell Biol. 27: 217-227.

Tartakoff, A.M. 1983. Perturbation of vesicular traffic with the carboxylic inophore monensin. Cell 32: 1026-1028.

Towbin, H., T. Staehelin, and J. Gordon. 1979. Electrophoretic transfer of protein from polyacrylamide gels to nitrocellulose sheets: Procedure and some applications Proc. Natl. Acad. Sci. 76: 4350-4354.

Valnes, K. and P. Brandtzaeg. 1985. Retardation of immunofluorescence fading during microscopy. $/$. Histochem. Cytochem. 33: 755-761.

von Heijne, G. 1981. On the hydrophobic nature of signal sequences. Eur. I. Biochem. 116: 419-422.

1985. Signal sequences: The limits of variation. J. Mol. Biol. 184: 99-105.

Walter, G. and R.F. Doolittle. 1983. Antibodies against synthetic peptides. In Genetic engineering: Principles and methods, (ed. J.K. Setlow and A. Hollaender), vol. 5, pp. 61-91. Plenum Press, New York. 


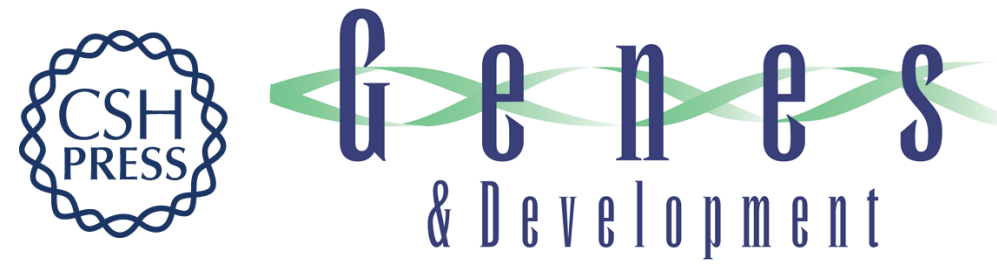

\section{Localization of the sea urchin Spec3 protein to cilia and Golgi complexes of embryonic ectoderm cells.}

E D Eldon, I C Montpetit, T Nguyen, et al.

Genes Dev. 1990, 4:

Access the most recent version at doi:10.1101/gad.4.1.111

References This article cites 38 articles, 15 of which can be accessed free at:

http://genesdev.cshlp.org/content/4/1/111.full.html\#ref-list-1

License

Email Alerting

Service

Receive free email alerts when new articles cite this article - sign up in the box at the top right corner of the article or click here.

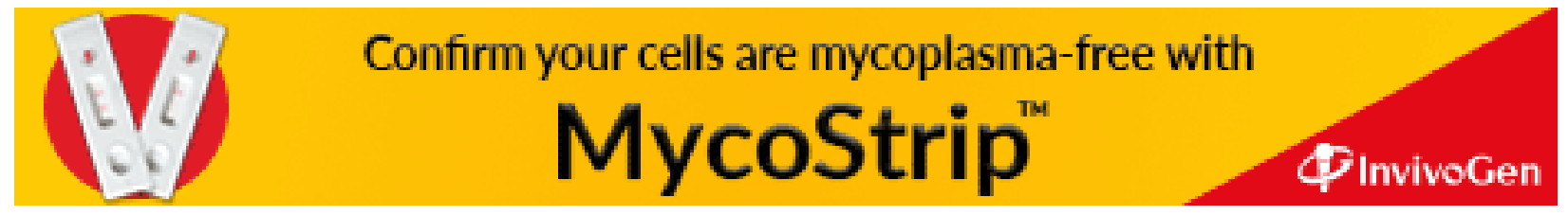

